\title{
Nucleoporin NUP153 guards genome integrity by promoting nuclear import of 53BP1
}

\author{
P Moudry ${ }^{1,2}$, C Lukas ${ }^{2}$, L Macurek ${ }^{1}$, B Neumann ${ }^{3}$, J-K Heriche ${ }^{3}$, R Pepperkok ${ }^{3}$, J Ellenberg ${ }^{3}$, Z Hodny ${ }^{1}$, J Lukas ${ }^{\star, 2}$ and J Bartek ${ }^{\star, 1,2,4}$
}

53BP1 is a mediator of DNA damage response (DDR) and a tumor suppressor whose accumulation on damaged chromatin promotes DNA repair and enhances DDR signaling. Using foci formation of 53BP1 as a readout in two human cell lines, we performed an siRNA-based functional high-content microscopy screen for modulators of cellular response to ionizing radiation (IR). Here, we provide the complete results of this screen as an information resource, and validate and functionally characterize one of the identified 'hits': a nuclear pore component NUP153 as a novel factor specifically required for 53BP1 nuclear import. Using a range of cell and molecular biology approaches including live-cell imaging, we show that knockdown of NUP153 prevents 53BP1, but not several other DDR factors, from entering the nuclei in the newly forming daughter cells. This translates into decreased IR-induced 53BP1 focus formation, delayed DNA repair and impaired cell survival after IR. In addition, NUP153 depletion exacerbates DNA damage caused by replication stress. Finally, we show that the C-terminal part of NUP153 is required for effective 53BP1 nuclear import, and that 53BP1 is imported to the nucleus through the NUP153-importin- $\beta$ interplay. Our data define the structure-function relationships within this emerging 53BP1-NUP153/importin- $\beta$ pathway and implicate this mechanism in the maintenance of genome integrity.

Cell Death and Differentiation (2012) 19, 798-807; doi:10.1038/cdd.2011.150; published online 11 November 2011

The cellular DNA damage response (DDR) machinery serves as a biological barrier against accumulation of genetic changes associated with aging, and guards against neurodegenerative and immunodeficiency disorders and cancer. ${ }^{1}$ The proximal DDR kinases ATM, ATR and DNA-PK have a central role in response to various DNA lesions including DNA double strand breaks (DSBs), in part by phosphorylating histone $\mathrm{H} 2 \mathrm{AX}(\gamma \mathrm{H} 2 \mathrm{AX})$ and its sensor MDC1, which in turn coordinates recruitment of signaling and repair factors to DNA damage foci. ${ }^{1-4}$ These foci include 53BP $1^{5,6}$ whose retention at the DSB-flanking chromatin requires histone ubiquitylation by E3-ubiquitin ligases RNF8, RNF168 and HERC2, SUMOylation by the PIAS4/UBC9 complex, and histone methylation by MMSET and SET8 methyltransferases. ${ }^{1-3,7}$ Apart from the role of 53BP1 in telomere maintenance, cell cycle checkpoints and DNA repair by non-homologous end joining of DSBs, ${ }^{2,3,5,6} 53 \mathrm{BP} 1$ is also involved in response to viruses, ${ }^{8}$ and contributes to replication stress responses by protecting under-replicated genomic loci in G1 phase of the cell cycle..$^{9,10}$

Trafficking across the nuclear membrane occurs through nuclear pore complexes (NPCs), large channels consisting of over 30 nucleoporins (NUPs). The central channel of NPC is filled with hydrophobic phenylalanine-glycine $(F G)$ repeats contained in many NUPs, which mediate mostly low-affinity interactions with nuclear transport factors. NUP153 is also an FG-repeat containing NUP, but contains a high-affinity site for importin- $\beta .{ }^{11,12}$ NUP153 localizes on the nucleoplasmic face of the nuclear pore and is required for assembly of the nuclear basket and import of a subset of nuclear proteins. ${ }^{13}$ NUP153 also facilitates nuclear export of mRNA and ribonucleoprotein particles $^{14,15}$ and may have additional functions within the nucleus. ${ }^{16-19}$

Here, we performed an siRNA screen for novel factors involved in ionizing radiation (IR)-induced DDR foci formation,

\footnotetext{
${ }^{1}$ Department of Genome Integrity, Institute of Molecular Genetics, v.v.i., Academy of Sciences of the Czech Republic, Prague CZ-142 20, Czech Republic; ${ }^{2}$ Institute of Cancer Biology and Centre for Genotoxic Stress Research, Danish Cancer Society, Copenhagen DK-2100, Denmark; ${ }^{3}$ European Molecular Biology Laboratory (EMBL), Heidelberg D-69117, Germany and ${ }^{4}$ Institute of Molecular and Translational Medicine, Palacky University, Olomouc CZ-775 15, Czech Republic

${ }^{*}$ Corresponding author: J Lukas or J Bartek, Institute of Cancer Biology and Centre for Genotoxic Stress Research, Danish Cancer Society, Strandboulevarden 49, Copenhagen DK-2100, Denmark. Tel: + 4535257310 or + 45 35257357; Fax: + 45 35257721; E-mail: jil@ cancer.dk or jb@ cancer.dk Keywords: DNA repair; siRNA screen; 53BP1 nuclear import; nucleoporin NUP153; cell survival; ionizing radiation

Abbreviations: 53BP1, p53-Binding Protein 1; ANAPC10, anaphase-promoting complex subunit 10; ANAPC4, anaphase-promoting complex subunit 4; APC/C, anaphase-promoting complex/cyclosome; APH, aphidicolin; ASB18, ankyrin repeat and SOCS box protein 18; ATM, ataxia telangiectasia mutated; ATR, ataxia telangiectasia and Rad3-related protein; ATXN7, ataxin-7; BRCA1, breast cancer type 1 susceptibility protein; DDR, DNA damage response; DNA-PK, DNA-dependent protein kinase; DSB, Double strand breaks ; ECS, elongin, Cullin, SOCS-box containing complex; FANCD2, Fanconi anemia group D2 protein; FG repeat, phenylalanine-glycine repeat; GFP, green fluorescent protein; $\gamma \mathrm{H} 2 \mathrm{AX}$, phosphorylated histone H2AX at serine 139; HeLa, human adenocarcinoma cell line; HERC2, HECT domain and RCC1-like domain-containing protein 2; IR, ionizing radiation; MAP3K3, mitogen-activated protein kinase kinase kinase 3; MDC1, mediator of DNA damage checkpoint protein 1; Mec1, Mitosis entry checkpoint protein 1; MMSET, multiple myeloma SET domain-containing protein; NBS1, Nijmegen breakage syndrome protein 1; NPC, nuclear pore complex; NUP, nucleoporin; NUP153, nucleoporin 153kDa protein; NUP84, nucleoporin 84kDa protein; NUP93, nucleoporin 93kDa protein; PARP, poly [ADP-ribose] polymerase; PIAS4, protein inhibitor of activated STAT protein 4; Rad51, DNA repair protein RAD51; Rad53, Serine/threonineprotein kinase RAD53; RNF8, RING finger protein 8; RNF168, RING finger protein 168; SAGA, Spt-Ada-Gcn5 acetyltransferase; SCF, Skp, Cullin, F-box containing complex; SEC13L1, SEC13-like protein 1; SET8, SET domain-containing protein 8; Slx5, synthetic lethal of unknown function protein 5; SIx8, synthetic lethal of unknown function protein 8; TopBP1, DNA topoisomerase 2-binding protein 1; U2OS, human osteosarcoma cell line; UBA1, ubiquitin-activating enzyme E1; UBC9, ubiquitinconjugating enzyme 9

Received 11.8.11; revised 03.10.11; accepted 04.10.11; Edited by G Melino; published online 11.11.11
} 
combining custom-designed siRNA microarrays with highcontent microscopy. ${ }^{20,21}$ We chose to validate and characterize one of the identified factors, NUP153, and document its requirement for nuclear localization of 53BP1. Intriguingly, 53BP1 emerged as thus far the only genome caretaker whose nuclear import requires NUP153 and we therefore set out to investigate the NUP153-53BP1 crosstalk and its significance for the DDR.

\section{Results}

NUP153 is involved in nuclear import of 53BP1. By knocking down 1346 genes involved in the ubiquitinproteasome system and genes encoding zinc-finger proteins in human U2OS and HeLa cell lines, respectively, we aimed at identifying novel factors required for proper assembly of 53BP1 into radiation-induced foci. The lists of all targeted genes and the respective scores obtained in the screen are presented in Supplementary Tables 1 and 2, and the lists of 10 top-scoring genes ('hits') in U2OS and HeLa cells are provided in Tables 1 and 2, respectively. The fact that the known DSB regulators MDC1, RNF8 and RNF168 scored prominently in either cell type support the biological relevance of both our overall screening strategy and the additional top-scoring hits (Tables 1 and 2). Although their precise roles within the DDR machinery remains to be elucidated, it is noteworthy that among the top-scoring hits are proteins implicated in signal transduction and regulatory pathways operating through protein modifications, such as ubiquitylation (ANAPC10 and ANAPC4 components of the APC/C ubiquitin ligase, UBA1 ubiquitin activating enzyme and ASB18 - a candidate substrate-recognition component of the SCF-like ECS ubiquitin ligase complex), phosphorylation (MAP3K3 kinase involved in modulation of several key transcription factors) and acetylation (ATXN7, a component of the SAGA acetyl transferase complex).

One of the strongest hits that scored in both cellular backgrounds was NUP153 (Tables 1 and 2), whose downregulation reduced nuclear accumulation of 53BP1 and impaired formation of 53BP1 foci in cells exposed to IR (Figure 1a). Data mining of a previous genome-wide screen analyzing spontaneous DDR foci ${ }^{20}$ identified only two additional genes among the 26 tested NPC components (NUP93 and SEC13L1) whose knockdown resulted in partially cytoplasmic 53BP1 in a fraction of cells (Supplementary Table 3). In the presented screen of U2OS cells, the impact of NUP153 knockdown on IR-induced 53BP1 focus formation was the most robust, almost as pronounced as the knockdown of MDC1 and RNF168, two known regulators of 53BP1 retention at damaged chromatin that have no known relationship to the nuclear pore and were used here as positive controls (Figure 1b). ${ }^{20}$

The suppression of 53BP1 nuclear accumulation and focus formation was validated by two independent siRNAs to NUP153 (Figures 1c and d), reproduced in HeLa cells (Supplementary Figure S1A) and observed up to $8 \mathrm{~h}$ after IR (Supplementary Figure S1B). Biochemical fractionation confirmed that although total levels of 53BP1 remained unchanged in NUP153-depleted cells, 53BP1 partially redistributed to the cytoplasm after NUP153 knockdown (Figure 1e). To look more closely at 53BP1 localization in NUP153-depleted cells, we acquired images on confocal microscope (Figure 1c). In the efficiently NUP153-depleted cells, 53BP1 localized in the cytoplasm and also around the nucleus, presumably in nuclear pores. The latter localization

Table 1 Screen for suppressors of IR-induced 53BP1 focus formation; 10 top-scoring genes - U2OS cell line

\begin{tabular}{|c|c|c|c|}
\hline Gene name & Gene symbol & Highest Z-score & Average Z-score \\
\hline Ring finger protein 168 & $R N F 168$ & -2.11 & -1.86 \\
\hline Ring finger protein 8 & RNF8 & -2.10 & -2.10 \\
\hline Mediator of DNA-damage checkpoint 1 & $M D C 1$ & -1.99 & -1.47 \\
\hline Nucleoporin $153 \mathrm{kDa}$ & NUP153 & -1.93 & -1.71 \\
\hline Ubiquitin-like modifier activating enzyme 1 & UBA1 & -1.92 & -1.55 \\
\hline Target of myb1 (chicken) & TOM1 & -1.80 & -1.33 \\
\hline Anaphase promoting complex subunit 10 & ANAPC10 & -1.79 & -1.17 \\
\hline Cyclin T2 & CCNT2 & -1.55 & -1.17 \\
\hline Ataxin 7 & ATXN7 & -1.54 & -1.19 \\
\hline Mitogen-activated protein kinase kinase kinase 3 & МАРЗКЗ & -1.54 & -1.03 \\
\hline
\end{tabular}

Table 2 Screen for suppressors of IR-induced 53BP1 focus formation; 10 top scoring genes - HeLa cell line

\begin{tabular}{|c|c|c|c|}
\hline Gene name & Gene symbol & Highest Z-score & Average Z-score \\
\hline $\begin{array}{l}\text { Ring finger protein } 168 \\
\text { Mediator of DNA-damage checkpoint } 1 \\
\text { Ring finger protein } 8 \\
\text { Nucleoporin } 153 \mathrm{kDa} \\
\text { Anaphase promoting complex subunit } 4 \\
\text { Golgi associated, gamma adaptin ear containing, ARF binding protein } 3 \\
\text { Cingulin } \\
\text { Ring finger protein } 121 \\
\text { BTB (POZ) domain containing } 2 \\
\text { Ankyrin repeat and SOCS box-containing } 18\end{array}$ & $\begin{array}{l}\text { RNF168 } \\
\text { MDC1 } \\
\text { RNF8 } \\
\text { NUP153 } \\
\text { ANAPC4 } \\
\text { GGA3 } \\
\text { CGN } \\
\text { RNF121 } \\
\text { BTBD2 } \\
\text { ASB18 }\end{array}$ & $\begin{array}{l}-2.22 \\
-1.88 \\
-1.50 \\
-1.34 \\
-1.15 \\
-0.93 \\
-0.92 \\
-0.78 \\
-0.75 \\
-0.72\end{array}$ & $\begin{array}{l}-0.50 \\
-0.66 \\
-1.07 \\
-0.97 \\
-0.76 \\
-0.59 \\
-0.80 \\
-0.66 \\
-0.74 \\
-0.60\end{array}$ \\
\hline
\end{tabular}


a

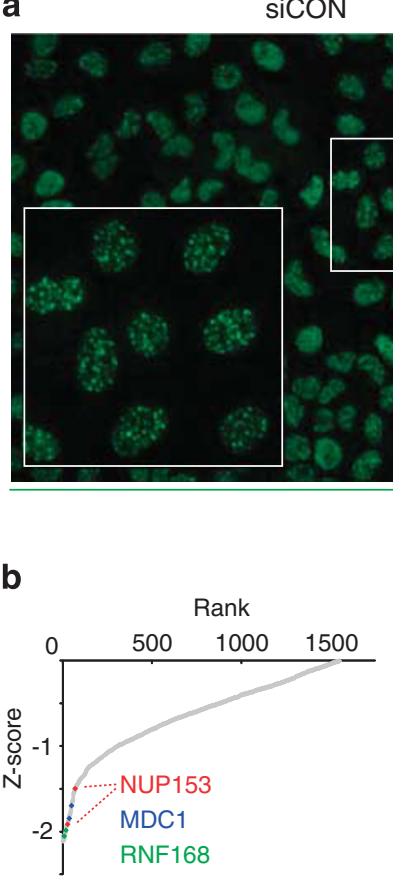

C
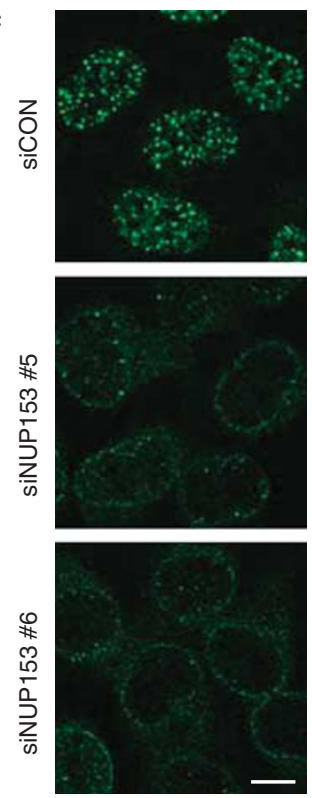

53BP1 d

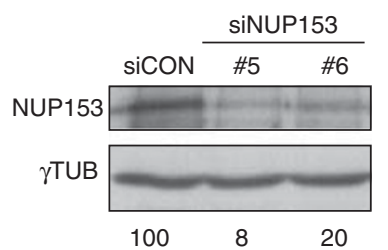

f
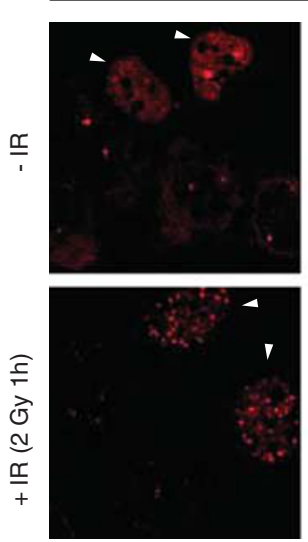

$53 \mathrm{BP} 1$
siNUP153 \#4

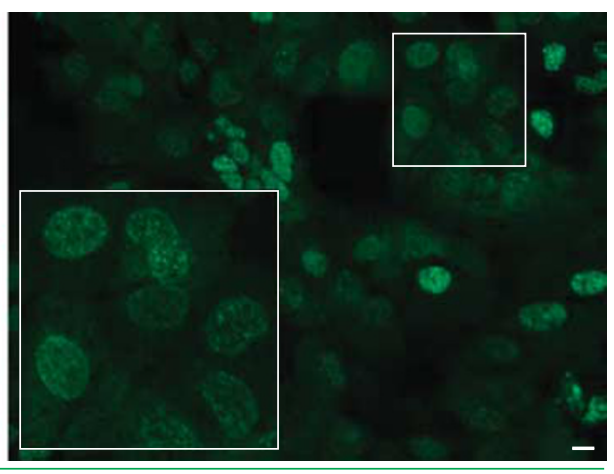

e $+\quad++\operatorname{sinUP153} \# 5$

WCE C N C N
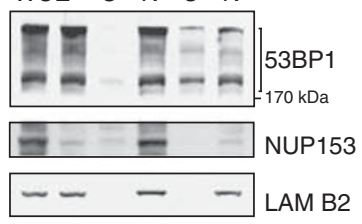

$\sim \sim-\quad \sim \alpha$ TUB

U2OS + siNUP153 \#5

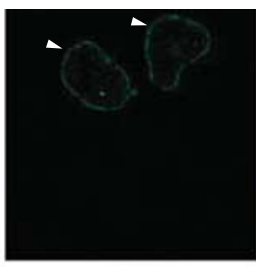

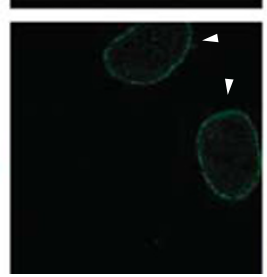

GFP-NUP153*
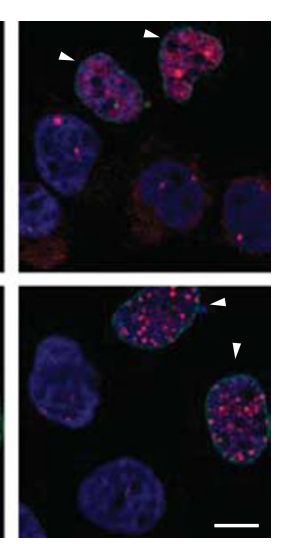

MERGE

Figure 1 NUP153 is required for efficient formation of 53BP1 foci. (a) Representative fields from the siRNA arrays showing U2OS cells grown on control or NUP153targeting siRNAs and immunostained for 53BP1 $1 \mathrm{~h}$ after 2 Gy of IR. (b) Scatter plot of Z-scores derived from the RNAi screen for 53BP1 foci formation factors in U2OS cells. The plotted values show the area of the decreased 53BP1 foci per cell. The positions of two NUP153 siRNAs are indicated (red). MDC1 (blue) and RNF168 (green) are positive controls. (c) U2OS cells were treated with the indicated siRNAs, incubated for 3 days, subjected to IR (2 Gy) and $1 \mathrm{~h}$ later immunostained for $53 \mathrm{BP} 1$. (d) Immunoblot of cell lysates from (c). Remaining protein levels of NUP153, normalized to loading control ( $\gamma$-tubulin), are indicated (bottom). (e) Cytosolic, nuclear and whole-cell extracts (C, N and WCE, respectively) were probed with indicated antibodies (see Materials and Methods for details of 53BP1 migration pattern). (f) U2OS cells were treated with the NUP153targeting siRNA (siNUP153 no. 5), transfected with the expression plasmid for RNAi-resistant GFP-NUP153*, irradiated as in (c) and immunostained for 53BP1. Nuclei were stained with DAPI. Arrowheads mark cells expressing GFP-NUP153*. Scale bars, $10 \mu \mathrm{m}$

pattern likely also explains residual amounts of 53BP1 in the nuclear fraction after NUP153 knockdown (Figure 1e), whereas the appearance of a sizable fraction of 53BP1 in the cytoplasmic fraction (Figure 1e) is consistent with the subcellular distribution observed by fluorescence microscopy. The partial distribution between the cytosolic and nuclear fractions seen by immunoblots may partly also reflect the fact that individual cells within the siRNA-treated population 
showed diverse extent of NUP153 depletion, with consequent variable impact on 53BP1 subcellular localization. In otherwise unperturbed cells, NUP153 knockdown selectively affected nuclear localization of 53BP1, with only modest effects on BRCA1 in subset of cells (Supplementary Figure S2A) and no effects on DDR factors, such as MDC1, NBS1, Rad51 and TopBP1. Finally, 53BP1 nuclear accumulation and formation of spontaneous and IR-induced 53BP1 foci were rescued by expression of siRNA-resistant GFP-NUP153*, confirming the specificity of the observed phenotype (Figure 1f).

Immunofluorescence analysis revealed that the IR-induced nuclear focus formation of BRCA1, MDC1, $\gamma \mathrm{H} 2 \mathrm{AX}$ and RNF168 was not affected by knockdown of NUP153, suggesting that loss of NUP153 specifically had an impact on the localization and foci formation of 53BP1 (Figure 2a). This result is consistent with a report showing that NUP153 knockdown does not impair global transport function of NPC. ${ }^{22}$ Time-lapse microscopy using a stable U2OS cell line expressing GFP-53BP1 ${ }^{10}$ uncovered that NUP153 knockdown impaired reentry of 53BP1 into the nucleus at the time of mitotic exit and reassembly of the nuclear envelope (Figure 2b). Although a fraction of 53BP1 eventually entered the nucleus, the bulk of 53BP1 remained in the cytoplasm for most of the cell cycle (our unpublished observation). Together, these data suggest that NUP153 is required for nuclear import of 53BP1.

\section{Knockdown of NUP153 delays DSB repair and impairs} cell survival. To test the significance of NUP153 knockdown on DNA repair, we exposed siRNA-treated cells to a moderate dose of IR (2Gy) and followed the MDC1 nuclear DSB foci in time. The number of nuclei with more than five MDC1 foci detected early (1 h) after IR was very similar in control, NUP153- and RNF168-deficient cells $(93 \%$, $91 \%$ and $98 \%$, respectively). However, although in control cells MDC1 progressively dissociated from DSBs (consistent with ongoing DNA repair), knockdown of NUP153 and

a

$\mathrm{U} 2 \mathrm{OS}+\operatorname{siRNA}+\mathrm{IR}(5$ Gy $1 \mathrm{~h})$
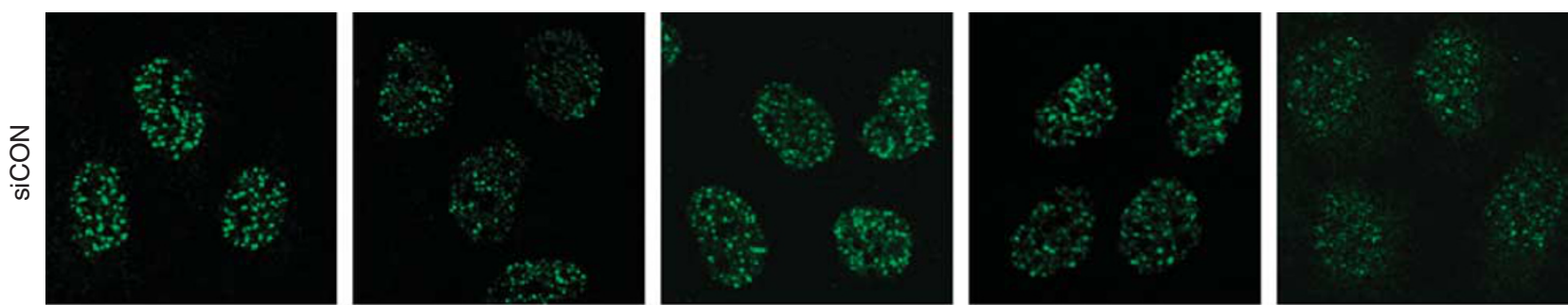

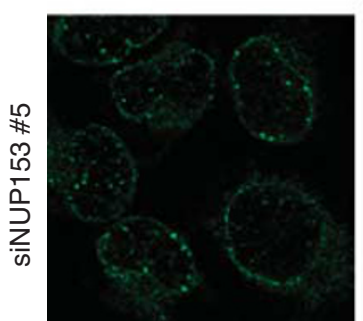

53BP1

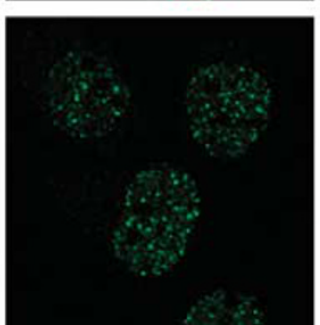

BRCA1

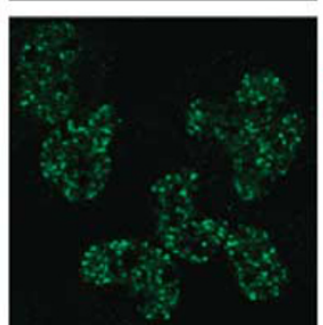

MDC1

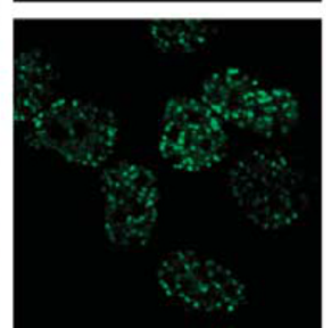

$\gamma \mathrm{H} 2 \mathrm{AX}$

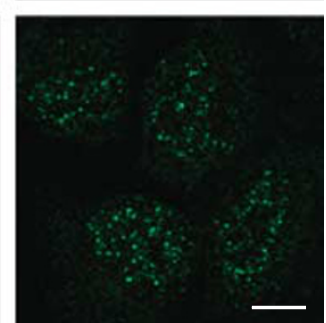

RNF168

b

U2OS GFP-53BP1
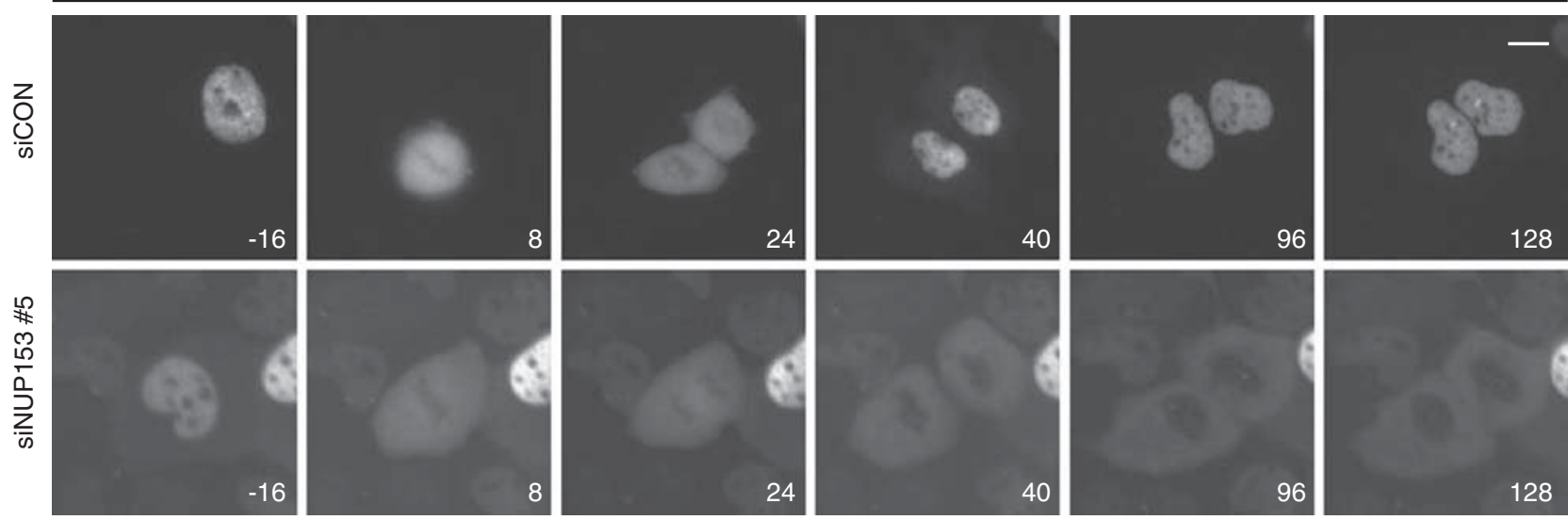

Time from initiation of mitosis (min)

Figure 2 NUP153 regulates nuclear import of 53BP1. (a) U2OS cells were treated with siRNAs for 3 days, irradiated (5 Gy) and $1 \mathrm{~h}$ later stained with indicated antibodies. (b) U2OS cells stably expressing GFP-53BP1 treated with indicated siRNAs were subjected to time-lapse microscopy. Scale bars, $10 \mu \mathrm{m}$ 
RNF168 (the upstream regulator of 53BP1 used here as a positive control) caused persistence of the MDC1 foci even at the time points when the control cells returned to the predamage values (Figures $3 a$ and $b$; and see Supplementary Figure S1C for siRNA efficiency). We detected only modest changes in the cell cycle profile in NUP153-silenced cells (Supplementary Figure S2B), indicating that indirect effects of cell cycle position were unlikely to account for the observed effects on DNA repair. Consistent with the impaired resolution of repair foci, we found that NUP153 knockdown impaired the long-term survival of U2OS cells exposed to IR (Figure 3c).

Depletion of NUP153 enhances DNA damage caused by replication stress. In addition to the delayed DSB repair and decreased survival after IR, depletion of NUP153 reduced the intensity of 53BP1 nuclear bodies in otherwise unstressed G1 cells (Supplementary Figure S1D). As these nuclear compartments have been associated with shielding DNA lesions arising from unresolved replication intermediates, ${ }^{9,10}$ we hypothesized that cytoplasmic sequestration of 53BP1 may also undermine cellular responses to replication stress. Indeed, NUP153-depleted cells in which 53BP1 was retained in the cytoplasm showed increased fraction of nuclei with elevated number of $\gamma \mathrm{H} 2 \mathrm{AX}$ foci after treatment with low doses of aphidicolin, an inhibitor of DNA polymerases $\alpha$ and $\delta$ (Figures $4 \mathrm{a}$ and $\mathrm{b}$ ), consistent with the notion that 53BP1 participates in protecting cells against replication stress. We note that NUP153 depletion reduced, but not completely eliminated, accumulation of 53BP1 in G1 nuclear bodies (Supplementary Figure S1D); it is therefore possible that additional factors whose nuclear

$\mathrm{U} 2 \mathrm{OS}+\mathrm{IR}(2 \mathrm{~Gy})$
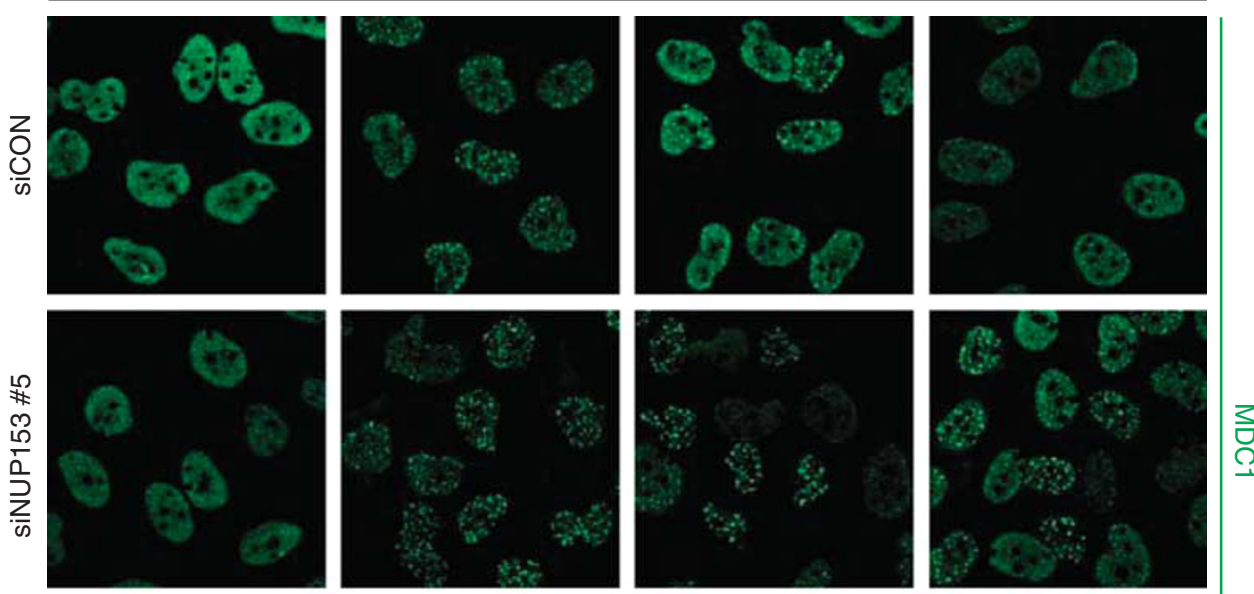

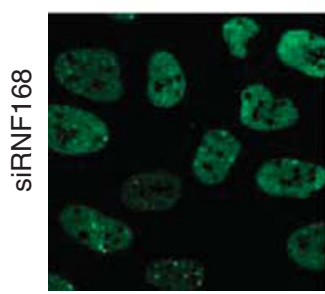

$\mathrm{Oh}$

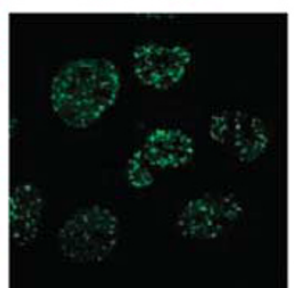

$1 \mathrm{~h}$

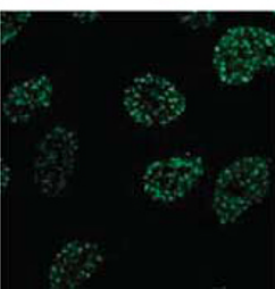

$4 \mathrm{~h}$

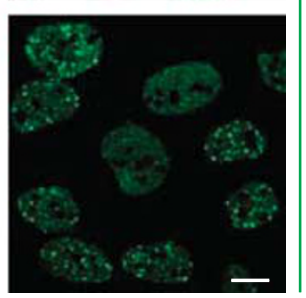

$8 \mathrm{~h}$ b

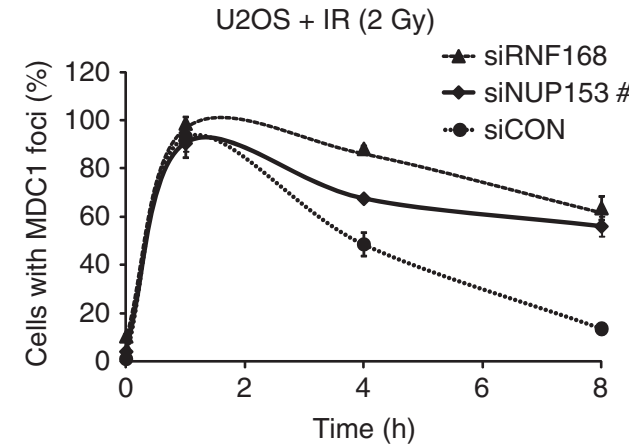

c

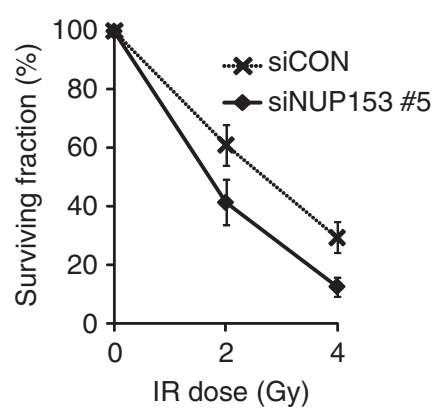

Figure 3 Depletion of NUP153 delays DNA repair and reduces survival after IR. (a) U2OS cells were treated with indicated siRNAs for 3 days and irradiated (2 Gy). At indicated times, cells were immunostained for MDC1. (b) Quantification of MDC1 foci from (a). The graph shows nuclei with $>5 \mathrm{MDC} 1$ foci (>500 cells were scored per time point, $n=2$ ). Error bars represent S.D. (c) U2OS cells treated with the indicated siRNAs for $72 \mathrm{~h}$, irradiated and plated. Colonies of $>50$ cells were scored 10 days later. Error bars represent S.D. $(n=2)$. Scale bar, $10 \mu \mathrm{m}$ 
a
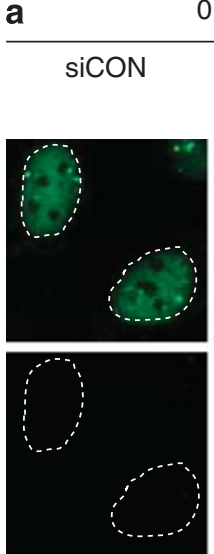

$0.2 \mu \mathrm{M}$ aphidicolin
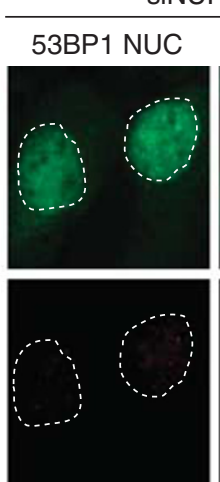

b

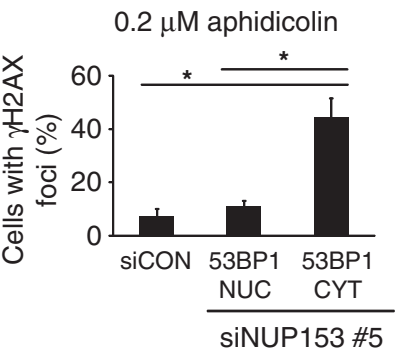

Figure 4 NUP153 depletion exacerbates DNA damage caused by replication stress. (a) U2OS cells were treated with indicated siRNAs for $72 \mathrm{~h}$, exposed to $0.2 \mu \mathrm{M}$ aphidicolin for $16 \mathrm{~h}$, stained with 53BP1 and $\gamma \mathrm{H} 2 \mathrm{AX}$, and counterstained with DAPI. Cells in G1 were selected based on total DAPI signal intensity (Olympus ScanR software, Olympus, Tokyo, Japan) and the siNUP153-treated cells divided into: 53BP1-nuclear and 53BP1-cytoplasmic according to their mean intensity of nuclear 53BP1 signal. (b) Cells from (a) were analyzed for $\gamma \mathrm{H} 2 \mathrm{AX}$ foci (cells with $>5 \mathrm{\gamma H} 2 \mathrm{AX}$ foci were scored as positive); 225 cells were analyzed in each category $\left({ }^{\star} P<0.02 ; n=3\right)$. Scale bar, $10 \mu \mathrm{m}$
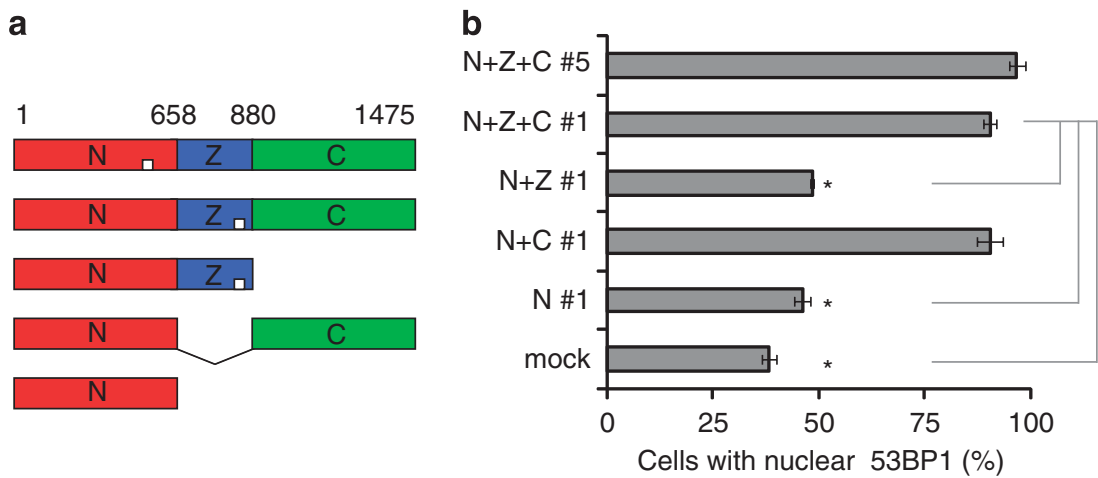

Figure 5 The C-terminus of NUP153 is required for nuclear import of 53BP1. (a) Domain structure of NUP153 and different siRNA resistant constructs; N: N-terminal domain, Z: zinc-finger region and C: C-terminal domain. White squares represent regions targeted with siRNA. (b) U2OS cells were treated with NUP153-targeting siRNAs (siNUP153 no. 5 or no. 1), transfected with indicated expression plasmids and $48 \mathrm{hlater}$ immunostained for 53BP1. The graph shows \% of transfected cells with nuclear 53BP1 $\left({ }^{*} P<0.001 ; n=3\right)$

import is regulated by NUP153 cooperate with 53BP1 to protect genomic loci affected by replication stress.

The C-terminus of NUP153 is required for 53BP1 nuclear import. To determine which domains of NUP153 are necessary for nuclear import of 53BP1, we performed a functional complementation experiment using full-length and previously described NUP153 mutants ${ }^{22}$ in which the C-terminal part containing the FG repeats, central zinc finger domain or both were deleted (Figure 5a). As reported, the $\mathrm{N}$-terminal part of NUP153 is required for incorporation into NPC. ${ }^{23}$ Among the tested NUP153 variants, only the fragment containing the intact $\mathrm{N}$ and $\mathrm{C}$ termini $(\mathrm{N}+\mathrm{C})$ was able to fully rescue 53BP1 nuclear accumulation in cells depleted of endogenous NUP153 (Figure 5b), indicating that the internal zinc-finger domain of NUP153 is not required for nuclear import of 53BP1. Instead, these experiments suggested that 53BP1 nuclear import could be mediated via the C-terminus of NUP153, whose FG repeats are known to interact with importin- $\beta .^{11,12}$
Disruption of importin- $\beta$ transport pathway blocks nuclear import of 53BP1. To test whether importin- $\beta$ is involved in 53BP1 nuclear import, we took advantage of previous reports showing that overexpression of the $\mathrm{N}$ terminal and C-terminal part of NUP153 inhibits transportin-1 and importin- $\beta$-mediated transport, respectively. ${ }^{24}$ We expressed these fragments in U2OS cells and assayed their impact on 53BP1 localization. Although the $\mathrm{N}$-terminal fragment of NUP153 did not affect 53BP1 localization, cells overexpressing the C-terminal fragment showed a predominantly cytoplasmic 53BP1 (Figure 6a). Consistent with published results, we observed interaction of the NUP153 C-terminal fragment with importin- $\beta$ (Supplementary Figure S3A) and also an interaction of endogenous NUP153 with importin- $\beta$ protein (Supplementary Figure S3B). Thus, overexpression of the C-terminal part of NUP153 appears to generate a dominant negative effect on nuclear import of 53BP1, indicating that accumulation of 53BP1 in the nucleus may rely on the importin- $\beta$ pathway.

Next, we asked to which extent the nuclear import of 53BP1 is dependent on importin- $\beta$ and transportin-1, respectively. 
a

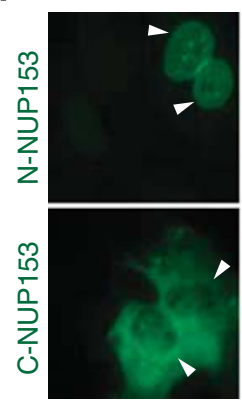

GFP
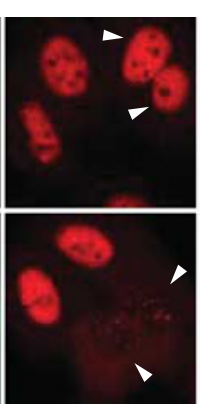

53BP1

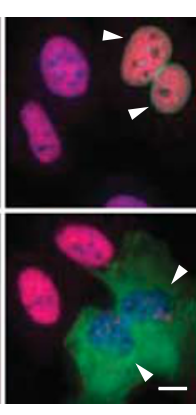

MERGE b

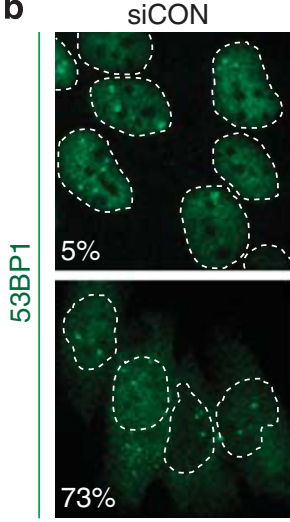

SiRAN
silMP $\beta$

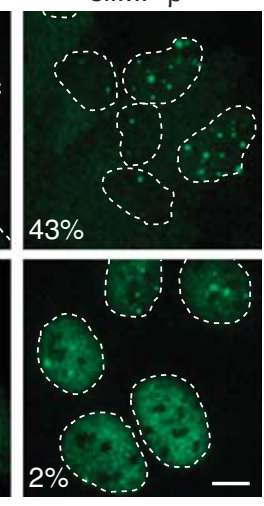

siTNPO1
C

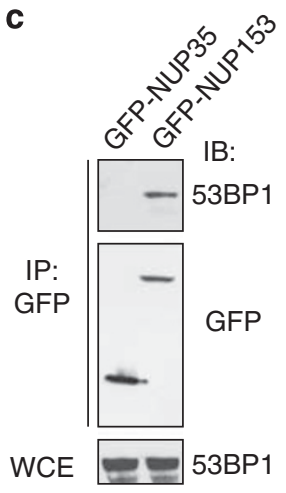

d

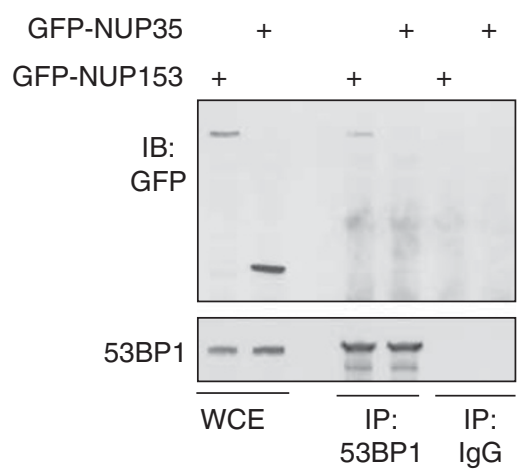

e

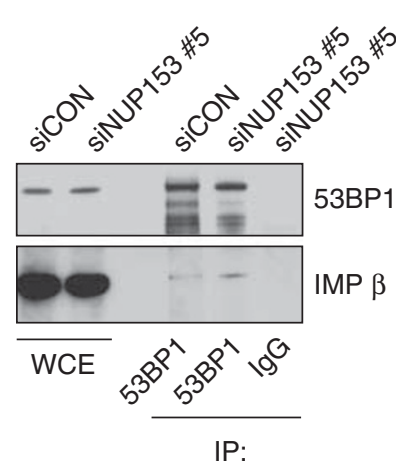

Figure 6 Disruption of importin- $\beta$ transport pathway blocks nuclear import of 53BP1. (a) U2OS cells were transfected with GFP tagged N-and C-terminal domains of NUP153 and $48 \mathrm{~h}$ later immunostained for 53BP1. Arrowheads mark the transfected cells. (b) U2OS cells were treated with indicated siRNAs and $72 \mathrm{~h}$ later immunostained for 53BP1; numbers in frames indicate the percentage of cells with detectable cytoplasmic 53BP1. (c) GFP was immunoprecipitated from U2OS transfected with GFP-NUP153 or GFP-NUP35 and samples were analyzed by immunoblotting with indicated antibodies. (d) 53BP1 was immunoprecipitated from U2OS cells transfected with GFP-NUP153 or GFP-NUP35 and samples were analyzed by immunoblotting with indicated antibodies. (e) 53BP1 was immunoprecipitated from cells treated with indicated siRNAs and immunoblotted with indicated antibodies. Scale bars, $10 \mu \mathrm{m}$

siRNA-mediated knockdown of importin- $\beta$ was relatively toxic, likely because importin- $\beta$ is essential for nuclear import of a large number of cargoes; ${ }^{25}$ nevertheless, its depletion resulted in nuclear exclusion of 53BP1 in a fraction of cells (Figure $6 \mathrm{~b}$ ) within the time frame of the experiment. In contrast, knockdown of transportin-1 had no effect on 53BP1 localization (Figure 6b). Nuclear import of proteins is also dependent on Ran GTPase. ${ }^{26}$ Indeed, knockdown of Ran resulted in a detectable cytoplasmic 53BP1 in the majority of cells, analogous to knockdown of importin- $\beta$ (Figure 6b). Efficiency of transportin-1 and importin- $\beta$ knockdown, respectively, was confirmed by immunoblotting (Supplementary Figure S3C).

Finally, we focused on potential physical interactions among the key proteins implicated by the above results in the nuclear import of 53BP1. Indeed, we were able to coimmunoprecipitate endogenous 53BP1 with GFP-NUP153, but not with a control unrelated NUP GFP-NUP35 (Figure 6c), and, vice versa, GFP-NUP153, but not GFP-NUP35, with endogenous 53BP1 (Figure 6d), suggesting specificity of the observed interaction. Moreover, we found an interaction between endogenous 53BP1 and endogenous importin- $\beta$ (Figure 6e). The latter interaction was detectable regardless of whether NUP153 was knocked down in the analyzed cells or not (Figure 6e), a result that is consistent with the current models of importin- $\beta$ forming its complexes with cargo proteins (here 53BP1) in the cytosol, prior to the interaction of such importin- $\beta /$ cargo complex with the NPC. ${ }^{27}$ These results further support the role of the NUP153/importin- $\beta$ pathway in nuclear import of 53BP1.

\section{Discussion}

The results presented in this article can contribute to the better understanding of the genome maintenance machinery in mammalian cells in two ways. First, we provide in full our data from a high-content siRNA-based microscopy screen to identify potential novel regulators of radiation-induced assembly of DDR factors at DSB sites, as monitored by 53BP1 focus formation in two human cancer cell lines. This data set (Supplementary Tables 1 and 2) complements other recent, genome-wide siRNA screens for factors involved in spontaneous $^{20}$ or radiation-induced ${ }^{28}$ foci formation by 53BP1, and we present these results as an information resource. Second, as discussed below in more detail, our results revealed a novel, hitherto unrecognized level of regulation of 53BP1: 
through the NUP153-mediated nuclear import as a pathway required for proper localization of 53BP1 and the maintenance of genome integrity.

From a wider perspective, our findings of the 53BP1NUP153/importin- $\beta$ pathway as an important aspect of the DDR network add to an emerging evidence of subcellular trafficking as an integral part of genome surveillance. Such evidence encompasses ATM-regulated nuclear export ${ }^{29}$ and import, $^{30}$ as well as the import of essential genome caretakers, such as the FANCD2, RAD51 or BRCA1 repair proteins, ${ }^{31-33}$ ribonucleotide reductases, ${ }^{34}$ DNA damageregulated transcription factors, ${ }^{35}$ the ATM-activator protein Aven $^{36}$ or p53 tumor suppressor. ${ }^{37}$

Although our overall understanding of the role(s) for mammalian NUPs and NPCs in genome maintenance is still in its infancy, significant advances in this research area have already been made based on experiments with yeast models. For example, damaged DNA was shown to be recruited for repair to nuclear pores through an interplay between the Nup84 complex and the SIx5/SIx8 SUMO-dependent ubiquitin ligase of the budding yeast. ${ }^{38}$ Most recently, the budding yeast checkpoint kinases Mec1 and Rad53 that are critical for the maintenance of genomic stability under DNA replication stress conditions were shown to target the nuclear porecoupled machinery responsible for mRNA export from actively transcribed genes. ${ }^{39}$ The topological tension created by NPCtethered mRNAs under replication stress becomes neutralized through phosphorylation of several inner-basket NUPs by the DDR kinases, thereby protecting the stability of replication forks and allowing recovery from such potentially hazardous conditions that could otherwise seriously undermine genome integrity. ${ }^{39}$ Although very inspiring, these emerging links between NUPs and various aspects of DNA damage checkpoints and repair appear to be distinct from the role discovered in our present study, of NUP153 in the nuclear import, and consequently the function of 53BP1 in DDR of mammalian cells.

In terms of a potential patho-physiological relevance of our present findings, it is noteworthy that the function of NUP153 was required for proper cellular responses to both DSBs (here induced by IR) and replication stress, and thereby for critical cell fate decisions including cell survival. This further indicates the significance of the 53BP1-NUP153/importin- $\beta$ pathway in tumorigenesis and response to genotoxic treatment modalities that are commonly used in clinical oncology. As oncogene-evoked replication stress is a feature shared by many transforming oncogenes, and 53BP1 foci form 'spontaneously' in such cellular models and early lesions of human tumors in vivo, ${ }^{40,41}$ the NUP153-53BP1 mechanism contributes to the DDR-mediated anti-cancer barrier. ${ }^{42}$ Furthermore, the significance of our present results for potential treatment outcome reflects the key role of 53BP1 in response to IR, but also to other treatment modalities, such as cisplatinbased chemotherapy or targeted treatment by inhibitors of PARP, another important DDR component, particularly in subset of tumors defective in the BRCA1/2 tumor suppressors and DNA repair factors. ${ }^{1}$ Two recent studies showed that BRCA1-deficient cancer cells can improve their overall genomic stability and even become resistant to cisplatin ${ }^{43}$ and PARP inhibitors ${ }^{44}$ by partial reversal of their defect in homologous recombination DNA repair, through loss of 53BP1. ${ }^{43,44}$ The level, and possibly also subcellular localization (regulated by NUP153), of 53BP1 might therefore serve as a useful predictive marker to guide clinical decisions about the choice of appropriate personalized treatment of subsets of cancer patients in the not too distant future.

Last but not least, a very intriguing feature of the 53BP1NUP153/importin- $\beta$ pathway described here is its selectivity, in contrast to nuclear import of other DDR factors that also assemble at DSB sites, such as MDC1 or NBS1, for example. Such a degree of specificity of the NUP153-mediated pathway for 53BP1 indicates that other proteins associated with DNA damage-modified chromatin require distinct mechanisms for nuclear import. It will be rewarding to dissect these mechanisms and assess their potential roles in preventing diseases associated with genome instability.

\section{Materials and Methods}

Screen for 53BP1 regulators. A custom-designed siRNA library (Ambion, Austin, TX, USA; silencer select siRNAs) comprising of 1346 targets involved in ubiquitin-proteasome system (see Supplementary Table 4 for used siRNA sequences), SUMO regulators and zinc-finger proteins were used as described. ${ }^{20}$ Briefly, U2OS or HeLa cells were seeded on the siRNA arrays, incubated for 3 days, irradiated with two Gy, immunostained $1 \mathrm{~h}$ later with an antibody to 53BP1 and analyzed for 53BP1 nuclear foci. Each gene was targeted by two independent siRNAs and only the cases where both siRNAs showed the same phenotype were considered as hits.

Image acquisition, image analysis and Z-score calculation. Image acquisition of the siRNA arrays was performed on a wide-field fluorescence microscope (Zeiss Axiovert 200, Zeiss, Jena, Germany) equipped with a motorizedstage, $\times 20$ air objective (PlanApochromat $20 \times 10.8$, Zeiss), CCD camera (Coolsnap HQ, Roper Scientific, Ottobrunn, Germany) operated by Metamorph software (version 6.2r6; Molecular Devices, Sunnyvale, CA, USA). The number of 53BP1 foci per nucleus was determined by automated routine using free software ImageJ (http://rsb.info.nih.gov/ij/). The nuclei were detected using the Hoechst signal, the average number of 53BP1 foci per nucleus was determined for each frame, and the values normalized to the average number of foci in nuclei exposed to the non-targeting controls within the given siRNA microarray. Impact of each siRNA on 53BP1 foci formation was determined according to its Z-score: $Z=(X-\mu) / \sigma$ (where $\mathrm{X}$ is the raw score, $\sigma$ is the S.D. of the population and $\mu$ is the mean of the population).

Cell culture. Human U2OS and HeLa cell lines were cultured in Dulbecco's Modified Eagle's medium supplemented with 10\% foetal bovine serum (Invitrogen, Carlsbad, CA, USA) and penicillin/streptomycin (Sigma-Aldrich, St.Louis, MO, USA) in a humidified atmosphere of $5 \% \mathrm{CO} 2$ at $37^{\circ} \mathrm{C}$

Plasmids and antibodies. The plasmid for GFP-NUP153 (pEGFP-NUP153) was described previously. ${ }^{17}$ To generate siRNA-insensitive GFP-NUP $153^{*}$ mutant, silent mutations were introduced into the siNUP153 no. 5 target sequence in the NUP153 coding region using the QuikChange II Site-Directed Mutagenesis Kit (Stratagene/Agilent Technologies, Santa Clara, CA, USA). Plasmid transfections were performed using FuGENE 6 (Roche, Mannheim, Germany) according to the manufacturer's instructions. Antibodies used in this study included mouse monoclonal antibodies to $\gamma \mathrm{H} 2 \mathrm{AX}$ (Millipore, Billerica, MA, USA), BRCA1, GFP (Santa Cruz Biotechnology, Santa Cruz, CA, USA), MDC1 (Sigma-Aldrich), NBS1 (GeneTex, Irvine, CA, USA) and NUP153 (Abcam, Cambridge, UK); rabbit polyclonal antibodies to 53BP1, RAD51, TopBP1 (Santa Cruz Biotechnology), 53BP1 (Millipore), MDC1 (Abcam) and RNF168; ${ }^{28}$ and goat polyclonal antibody to $\gamma$-tubulin (Santa Cruz Biotechnology). Note that some but not all antibodies to 53BP1 recognize a triplet of bands on western blots (see Figure 1e) that likely represent modified versions of the protein. Importantly, the cellular staining signal obtained with these antibodies selectively disappeared after siRNA-mediated knockdown of 53BP1 (data not shown), also supporting the notion that the three western blot bands represent modified 53BP1. 
RNA interference. All transient siRNA transfections were performed using Lipofectamine RNAiMAX (Invitrogen) and oligonucleotides with siRNA duplexes purchased from Ambion. siRNA used: negative control siRNA no. 1 (s229084), siNUP153 no. $1,{ }^{22}$ siNUP153 no. 4 (s19374), siNUP153 no. 5 (s19375), siNUP153 no. 6 (s19376), siRNF168 (ID no. 126171), silMP- $\beta$ (s7919), siTNP01 (s7933) and siRan (ID no. 120462).

Immunofluorescence and immunoblotting. Cells cultured on glass coverslips were fixed in $4 \%$ formaldehyde for $15 \mathrm{~min}$ at RT, permeabilized 5 min with $0.2 \%$ (v/v) Triton X-100 in PBS and incubated with primary antibodies for $60 \mathrm{~min}$ at RT. Following washing step, the coverslips were incubated with goat anti-rabbit or goat antimouse Alexa Fluor 488 or Alexa Fluor 568 (Invitrogen) secondary antibodies for $60 \mathrm{~min}$ at RT and mounted using Vectashield mounting reagent with DAPI (Vector Laboratories, Burlingame, CA, USA). Image acquisition was done using a confocal microscope LSM510 (Zeiss) as described previously. ${ }^{10,20}$ The analysis of cell lysates by gel electrophoresis under denaturing conditions, followed by immunoblotting was performed as described. ${ }^{45}$ Briefly, whole cell extracts were separated by 6 or $10 \%$ SDS-PAGE and transferred to nitrocellulose membranes (GE Healthcare, Little Chalfont, UK). The membranes were blocked with $5 \%(\mathrm{w} / \mathrm{v})$ dry milk in $0.1 \%(\mathrm{v} / \mathrm{v})$ Tween-20 in PBS and probed with the primary antibodies listed above, followed by HRP-labeled secondary antibodies (Vector Laboratories and Santa Cruz Biotechnology) and visualized using ECL detection reagents (GE Healthcare).

Clonogenic survival assay. Sensitivity to IR was determined by transfecting U2OS cells with control and NUP153-targeting siRNA for $72 \mathrm{~h}$ followed by irradiation ( 2 and 4 Gy) and plating in increasing cell densities on a six-well plate. Colonies were allowed to grow for 10 days, fixed in $70 \%$ ethanol and stained with $1 \%$ crystal violet in ethanol. Colonies of $>50$ cells were counted, and the surviving fraction was calculated and normalized to untreated control.

Cellular fractionation. Cytoplasmic proteins were extracted from U2OS cells by incubating in buffer A ( $1 \mathrm{mM}$ HEPES, $10 \mathrm{mM} \mathrm{KCl}, 1.5 \mathrm{mM} \mathrm{MgCl}, 0.5 \mathrm{mM}$ DTT, $0.1 \%$ Triton $\mathrm{X}-100$ ) at $4^{\circ} \mathrm{C}$ for $10 \mathrm{~min}$ and spinning down at $5000 \times \mathrm{g}$ for $2 \mathrm{~min}$ at $4^{\circ} \mathrm{C}$. Nuclear fraction was obtained by extraction of remaining pellet in SDS sample buffer.

Immunoprecipitation. Cells were subjected to mild sonication in ice-cold extraction buffer (PBS pH 7.4, 1\% Triton X-100, 2 mM EDTA, 1 mM EGTA, $0.5 \mathrm{mM}$ DTT, $1 \mathrm{mM} \mathrm{NaF}, 25 \mathrm{mM} \beta$-glycerolphosphate, $1 \mathrm{mM} \mathrm{Na}_{3} \mathrm{VO}_{4}$, protease inhibitors). Cell extracts were clarified by 10 min centrifugation $20000 \times g$ at $4^{\circ} \mathrm{C}$. Antibodies were bound to protein A/G resin (Thermo Fischer Scientific, Rockford, IL, USA) and incubated with normalized cell extracts at $4^{\circ} \mathrm{C}$ for $3 \mathrm{~h}$. Immunoprecipitates were extensively washed and eluted by SDS sample buffer.

\section{Conflict of Interest}

The authors declare no conflict of interest.

Acknowledgements. We thank Dr. Ullman (University of Utah) and Dr. Durocher (University of Toronto) for reagents. This study was supported by Grant Agency of the Czech Republic (301/08/0353, P301/10/1525 and P305/10/P420), Institutional Grant (AVOZ5039906), the Danish Cancer Society, the Danish National Research Foundation, Novo Nordisk (R153-A12997), the Lundbeck Foundation (R-44-A4400) and the European Commission (projects TRIREME, Infla-Care, DDResponse and CZ.1.05/2.1.00/01.0030). PM was supported by Charles University in Prague.

All original images from the screens are available at Mitocheck website (http://mitocheck.org/).

1. Jackson SP, Bartek J. The DNA-damage response in human biology and disease. Nature 2009; 461: 1071-1078.

2. Ciccia A, Elledge SJ. The DNA damage response: making it safe to play with knives. $\mathrm{Mol}$ Cell 2010; 40: 179-204.

3. Lukas J, Lukas C, Bartek J. More than just a focus: the chromatin response to DNA damage and its role in genome integrity maintenance. Nat Cell Biol 2011; 13: 1161-1169.

4. Nakamura AJ, Rao VA, Pommier Y, Bonner WM. The complexity of phosphorylated H2AX foci formation and DNA repair assembly at DNA double-strand breaks. Cell Cycle 2010; 9 : 389-397.
5. DiTullio Jr RA, Mochan TA, Venere M, Bartkova J, Sehested M, Bartek J et al. 53BP1 functions in an ATM-dependent checkpoint pathway that is constitutively activated in human cancer. Nat Cell Biol 2002; 4: 998-1002.

6. Schultz LB, Chehab NH, Malikzay A, Halazonetis TD. p53 binding protein 1 (53BP1) is an early participant in the cellular response to DNA double-strand breaks. J Cell Biol 2000; 151: $1381-1390$.

7. Devgan SS, Sanal O, Doil C, Nakamura K, Nahas SA, Pettijohn K et al. Homozygous deficiency of ubiquitin-ligase ring-finger protein RNF168 mimics the radiosensitivity syndrome of ataxia-telangiectasia. Cell Death Differ 2011; 18: 1500-1506.

8. Perfettini JL, Nardacci R, Seror C, Raza SQ, Sepe S, Saidi H et al. 53BP1 represses mitotic catastrophe in syncytia elicited by the HIV-1 envelope. Cell Death Differ 2010; 17 : 811-820.

9. Harrigan JA, Belotserkovskaya R, Coates J, Dimitrova DS, Polo SE, Bradshaw CR et al. Replication stress induces 53BP1-containing OPT domains in G1 cells. J Cell Biol 2011; 193: 97-108.

10. Lukas C, Savic V, Bekker-Jensen S, Doil C, Neumann B, Pedersen RS et al. 53BP1 nuclear bodies form around DNA lesions generated by mitotic transmission of chromosomes under replication stress. Nat Cell Biol 2011; 13: 243-253.

11. Ben-Efraim I, Gerace L. Gradient of increasing affinity of importin beta for nucleoporins along the pathway of nuclear import. J Cell Biol 2001; 152: 411-417.

12. Otsuka S, Iwasaka S, Yoneda Y, Takeyasu K, Yoshimura SH. Individual binding pockets of importin-beta for FG-nucleoporins have different binding properties and different sensitivities to RanGTP. Proc Natl Acad Sci USA 2008; 105: 16101-16106.

13. Walther TC, Fornerod M, Pickersgill H, Goldberg M, Allen TD, Mattaj IW. The nucleoporin Nup153 is required for nuclear pore basket formation, nuclear pore complex anchoring and import of a subset of nuclear proteins. EMBO J 2001; 20: 5703-5714.

14. Bastos R, Lin A, Enarson M, Burke B. Targeting and function in mRNA export of nuclear pore complex protein Nup153. J Cell Biol 1996; 134: 1141-1156.

15. Soop T, Ivarsson B, Bjorkroth B, Fomproix N, Masich S, Cordes VC et al. Nup153 affects entry of messenger and ribosomal ribonucleoproteins into the nuclear basket during export. Mol Biol Cell 2005; 16: 5610-5620.

16. Antonin W, Ellenberg J, Dultz E. Nuclear pore complex assembly through the cell cycle: regulation and membrane organization. FEBS Lett 2008; 582: 2004-2016.

17. Daigle N, Beaudouin J, Hartnell L, Imreh G, Hallberg E, Lippincott-Schwartz J et al. Nuclear pore complexes form immobile networks and have a very low turnover in live mammalian cells. J Cell Biol 2001; 154: 71-84.

18. Rabut G, Doye V, Ellenberg J. Mapping the dynamic organization of the nuclear pore complex inside single living cells. Nat Cell Biol 2004; 6: 1114-1121.

19. Sukegawa J, Blobel G. A nuclear pore complex protein that contains zinc finger motifs, binds DNA, and faces the nucleoplasm. Cell 1993; 72: 29-38.

20. Doil C, Mailand N, Bekker-Jensen S, Menard P, Larsen DH, Pepperkok R et al. RNF168 binds and amplifies ubiquitin conjugates on damaged chromosomes to allow accumulation of repair proteins. Cell 2009; 136: 435-446.

21. Neumann B, Walter T, Heriche JK, Bulkescher J, Erfle H, Conrad C et al. Phenotypic profiling of the human genome by time-lapse microscopy reveals cell division genes. Nature 2010; 464: 721-727.

22. Mackay DR, Elgort SW, Ullman KS. The nucleoporin Nup153 has separable roles in both early mitotic progression and the resolution of mitosis. Mol Biol Cell 2009; 20: 1652-1660.

23. Enarson $P$, Enarson $M$, Bastos $R$, Burke $B$. Amino-terminal sequences that direct nucleoporin nup153 to the inner surface of the nuclear envelope. Chromosoma 1998; 107 : 228-236.

24. Shah S, Forbes DJ. Separate nuclear import pathways converge on the nucleoporin Nup153 and can be dissected with dominant-negative inhibitors. Curr Biol 1998; 8: $1376-1386$.

25. Frankel MB, Knoll LJ. The ins and outs of nuclear trafficking: unusual aspects in apicomplexan parasites. DNA Cell Biol 2009; 28: 277-284.

26. Izaurralde E, Kutay U, von Kobbe C, Mattaj IW, Gorlich D. The asymmetric distribution of the constituents of the Ran system is essential for transport into and out of the nucleus. EMBO J 1997; 16: 6535-6547.

27. Stewart M. Molecular mechanism of the nuclear protein import cycle. Nat Rev Mol Cell Biol 2007; 8: 195-208.

28. Stewart GS, Panier S, Townsend K, Al-Hakim AK, Kolas NK, Miller ES et al. The RIDDLE syndrome protein mediates a ubiquitin-dependent signaling cascade at sites of DNA damage. Cell 2009; 136: 420-434.

29. Miyamoto S. Nuclear initiated NF-kappaB signaling: NEMO and ATM take center stage. Cell Res 2011; 21: 116-130.

30. Pawlikowska P, Leray I, de Laval B, Guihard S, Kumar R, Rosselli F et al. ATM-dependent expression of IEX-1 controls nuclear accumulation of Mcl-1 and the DNA damage response. Cell Death Differ 2010; 17: 1739-1750.

31. Gildemeister OS, Sage JM, Knight KL. Cellular redistribution of Rad51 in response to DNA damage: novel role for Rad51C. J Biol Chem 2009; 284: 31945-31952.

32. Thompson ME. BRCA1 16 years later: nuclear import and export processes. FEBS J 2010; 277: 3072-3078.

33. Wang J, Sarkar TR, Zhou M, Sharan S, Ritt DA, Veenstra TD et al. CCAAT/enhancer binding protein delta (C/EBPdelta, CEBPD)-mediated nuclear import of FANCD2 by IPO4 augments cellular response to DNA damage. Proc Natl Acad Sci USA 2002; 107: 16131-16136. 
34. Lee YD, Wang J, Stubbe J, Elledge SJ. Dif1 is a DNA-damage-regulated facilitator of nuclear import for ribonucleotide reductase. Mol Cell 2008; 32: 70-80.

35. Fensgard $\mathrm{O}$, Kassahun $\mathrm{H}$, Bombik I, Rognes T, Lindvall JM, Nilsen H. A two-tiered compensatory response to loss of DNA repair modulates aging and stress response pathways. Aging (Albany, NY) 2010; 2: 133-159

36. Esmaili AM, Johnson EL, Thaivalappil SS, Kuhn HM, Kornbluth S, Irusta PM. Regulation of the ATM-activator protein Aven by CRM1-dependent nuclear export. Cell Cycle 2010; 9: 3913-3920.

37. Marchenko ND, Hanel W, Li D, Becker K, Reich N, Moll UM. Stress-mediated nuclea stabilization of p53 is regulated by ubiquitination and importin-alpha3 binding. Cell Death Differ 2010; 17: 255-267.

38. Nagai S, Dubrana K, Tsai-Pflugfelder M, Davidson MB, Roberts TM, Brown GW et al. Functional targeting of DNA damage to a nuclear pore-associated SUMO-dependent ubiquitin ligase. Science 2008; 322: 597-602.

39. Bermejo R, Capra T, Jossen R, Colosio A, Frattini C, Carotenuto W et al. The replication checkpoint protects fork stability by releasing transcribed genes from nuclear pores. Cell 2011; 146: 233-246.
40. Bartkova J, Horejsi Z, Koed K, Kramer A, Tort F, Zieger K et al. DNA damage response as a candidate anti-cancer barrier in early human tumorigenesis. Nature 2005; 434: 864-870.

41. Gorgoulis VG, Vassiliou LV, Karakaidos P, Zacharatos P, Kotsinas A, Liloglou T et al. Activation of the DNA damage checkpoint and genomic instability in human precancerous lesions. Nature 2005; 434: 907-913.

42. Halazonetis TD, Gorgoulis VG, Bartek J. An oncogene-induced DNA damage model for cancer development. Science 2008; 319: 1352-1355.

43. Bouwman P, Aly A, Escandell JM, Pieterse M, Bartkova J, van der Gulden $\mathrm{H}$ et al. 53BP1 loss rescues BRCA1 deficiency and is associated with triple-negative and BRCA-mutated breast cancers. Nat Struct Mol Biol 2010; 17: 688-695.

44. Bunting SF, Callen E, Wong N, Chen HT, Polato F, Gunn A et al. 53BP1 inhibits homologous recombination in Brca1-deficient cells by blocking resection of DNA breaks. Cell 2010; 141: 243-254.

45. Jensen SS, Madsen MW, Lukas J, Binderup L, Bartek J. Inhibitory effects of 1alpha, 25-dihydroxyvitamin $\mathrm{D}(3)$ on the G(1)-S phase-controlling machinery. Mol Endocrinol 2001; 15: $1370-1380$.

Supplementary Information accompanies the paper on Cell Death and Differentiation website (http://www.nature.com/cdd) 